\title{
MOOC COMO DISRUPCIÓN EDUCATIVA. PROPUESTAS DE MEJORA A PARTIR DEL ANÁLISIS DE UNA WEB*
}

\section{MOOC AS AN EDUCATIONAL DISRUPTION. PROPOSALS FOR IMPROVEMENT FROM THE ANALYSIS OF A WEBSITE}

https://doi.org/10.15304/ie.30.6467

\author{
Alfonso Chaves-Montero \\ Universidad de Huelva (España) \\ alfonso.chaves@dstso.uhu.es \\ Celia Corchuelo Fernández \\ Universidad de Huelva (España) \\ celia.corchuelo@dedu.uhu.es \\ Carmen María Aránzazu Cejudo Cortés \\ Universidad de Huelva (España) \\ carmen.cejudo@dedu.uhu.es \\ Walter Federico Gadea Aiello \\ Universidad de Huelva (España) \\ walter.gadea@,ddcc.uhu.es
}

\section{RESUMEN}

En este período de tiempo transcurrido desde que surgieron los MOOC se ha producido una gran difusión y evolución que los cursos actuales. La diversidad de tipos conduce a que se establezcan diferencias entre ellos en términos tales como la interacción entre los participantes, la propia estructura de los cursos, las formas de evaluar los conocimientos adquiridos o los objetivos de la enseñanza-aprendizaje. Esta investigación intenta analizar el por qué de la alta tasa de abandono de los cursos MOOC en España que allí se ofrecen, específicamente de la sección Educación y Enseñanza. Para ello, se pretende realizar un estudio exploratorio sobre los cursos y llevar a cabo una codificación en función de sus categorías profesionales, para posteriormente poder analizar en profundidad dichas categorías más afines a la educación. Por ello, se han visualizado los cursos

Recibido: 17/XII/2019. Aceptado: X/2020

* Investigación incluida en el Programa de la convocatoria de XX Proyectos de innovación docente 2018-2019 de la Universidad de Huelva. 
MOOC desde una perspectiva global y después desglosarlo para observar sus inconvenientes y las propuestas de mejora que deben hacerse. Finalmente, se describen los principales hallazgos de nuestra investigación como posibles mejoras de los MOOC en España.

Palabras clave: MOOC, disrupción educativa, propuestas de mejora, abandono escolar.

\begin{abstract}
In this period of time since the MOOC emerged, there has been a greater spread and evolution than the current courses. The diversity of types leads to differences between them in terms such as the interaction between participants, the structure of the courses themselves, ways of assessing the knowledge acquired or the objectives of the teaching-learning. This research aims to carry out an exploratory study on MOOC courses in Spain and to carry out a codification according to their professional categories, in order to later be able to analyse these categories more closely related to education. To this end, MOOC courses have been viewed from a global perspective and then broken down to observe their advantages and disadvantages and the proposals for improvement that need to be made. Finally, the main findings of our research are described as possible improvements to the MOOC in Spain.
\end{abstract}

Keywords: MOOC, educational disruption, proposals for improvement, school dropouts.

\title{
1. INTRODUCCIÓN Y ESTADO DE LA CUESTIÓN
}

El presente artículo es el resultado de una investigación que tiene como objetivo central realizar un estudio reflexivo y exploratorio acerca de cómo se pueden clasificar los cursos MOOC en función de las distintas categorías profesionales a las que éstos atienden.

Según Lane (2012), los MOOC se clasifican: por su finalidad, su forma y su temática. Si nos referimos a su finalidad, estamos hablando de qué objetivos persiguen los MOOC, fundamentalmente, la resolución de problemas prácticos y técnicos. Si hablamos de la segunda dimensión de la clasificación, se apela a su forma de realización, por ejemplo, si se basan en redes o trabajos colaborativos, etc. En referencia a la temática, tiene que ver con los temas y contenidos que desarrollan los MOOC.

El artículo desarrolla, dentro de las Universidades de España y de la diversidad de las temáticas desarrolladas en las distintas plataformas de enseñanza, un intento de clasificar la diversidad de temáticas realizadas, sus alcances, su distribución y su finalidad. Se intenta mostrar la dificultad o facilidad para su acceso, sus objetivos y sus áreas prioritarias de interés. El contenido del análisis permite comprender los potenciales de estos cursos y sus diversos temas y, nos da una perspectiva de aquellas temáticas que aún no se han desarrollado dentro de los distintos nichos de las Universidades de España.

La investigación parte del objetivo principal de indagar el motivo por el que existe una falta de interés del alumnado que participa en los cursos MOOC al comienzo del mismo, profundizando, en particular, en la categoría «Educación y enseñanza».

Para ello se han considerado los siguientes objetivos específicos: 
- Analizar cuantitativamente las categorías asociadas a la página web para determinar la situación.

- Analizar cuantitativamente y cualitativamente la categoría afín.

- Resaltar las ventajas e inconvenientes de la página web y si están condicionadas por el idioma y la plataforma.

- Determinar las propuestas de mejora más acordes para el alumnado que accede a los cursos MOOC.

\section{REVISIÓN DE LA LITERATURA}

\subsection{Antecedentes}

Desde sus inicios la educación ha ido progresando en función del sistema educativo al que pertenecía. Una educación que partía de un desarrollo social para mejorar la construcción y el conocimiento en todas sus virtudes, a partir de una serie de canales como las tecnologías de la información y la comunicación (TIC) (Dans, 2009; Chiappe Hine y Martínez Silva, 2015).

Una educación abierta bajo unos principios del software libre que en los años 70 y 80 han ayudado al reciente Movimiento Educativo Abierto de acuerdo a unas premisas dentro de su marco conceptual (Baranjuk, 2007; D’Antoni, 2009; Chiappe et al., 2015).

La formación continua, ha supuesto un cambio y un hito en este siglo XXI con la llegada de los MOOC, que dibuja un panorama educativo donde se ponen de relieve diferentes retos en el proceso de enseñanza y aprendizaje, debido, en gran parte, a sus características de masividad, ubicuidad y gratuidad que veremos más adelante.

En consecuencia, muchos investigadores intentan ahondar en los estudiantes sobre la forma en la cual la tecnología les va a facilitar las continuas demandas de la sociedad y que en su manera de evaluar debe verse reflejada de acuerdo a la Educación Superior y en los entornos de aprendizaje en línea (Earl, 2013; Gallego-Arrufat, Gamiz-Sanchez y Gutiérrez-Santiuste, 2015), que serán desarrollados en función de los sistemas educativos.

En la actualidad se ven amenazados por este tipo de metodología y en la cual en algunos de los casos se preguntan si el profesorado va a ser sustituido porque ya no imparte clases y el alumnado estudiará en casa (Grossman, 2013). Pero no es una amenaza, ni un sustituto de las clases presenciales, más bien, como una actividad extraescolar para reforzar las asignaturas tradicionales (Luján, 2013).

La actualidad, en cuanto a la globalización, la universalización de la comunicación, del conocimiento y de la cultura, y la formación a lo largo de toda la vida, dan un gran auge a la extensión de los MOOC (Gómez Galán, 2014; Silva, 2014; Calvo Salvador, Rodríguez Hoyos y Fernández Díaz, 2016) en sus diferentes vertientes educativas. 
Recientes investigaciones muestran a los MOOC como un nuevo y polémico fenómeno educativo con diferentes discrepancias entre las interacciones entre estudiante/profesor, estudiante/estudiante y la calidad de las prácticas educativas (Marcelo y Zapata, 2008; Jung, 2011; Chiappe et al., 2015) y su auge investigativo despierta un interés sobre este fenómeno de la nueva educación a distancia.

Es en España donde se han desarrollado unos cursos MOOC de forma más rápida y con vinculaciones a instituciones de educación superior para dar más facilidades a las ofertas educativas con especializaciones (Calvo et al., 2016) y que se desarrollarán a través de las diferentes plataformas.

\subsection{MOOC: Desde su conceptualización hasta su disrupción educativa}

Los Massive Online Open Courses (MOOC) comenzaron en 2008 de la mano de Siemens y Downes, que introdujeron el carácter masivo a los cursos abiertos en línea (SánchezVera, León-Urrutia y Davis, 2015) y empezaron a florecer en 2011 con su origen en el concepto de Social Learning basado en la posibilidad de aprender mediante la interacción con otras personas (Vivar, Vinader y Abuín, 2015). Los MOOC aparecieron en la formación superior para ser uno de los mayores acontecimientos disruptivos de los últimos años (Pappano, 2012; Anderson, 2013; Conole, 2013; Little, 2013; Aguaded, 2013; Aguaded, Vázquez Cano y López Meneses, 2016).

Bien es cierto que su paso por Europa está siendo más lento que por EEUU como elemento de disrupción en innovación educativa (Gaebel, 2013; Sancho, Oliver y Gisbert, 2015), y fue en 2012 cuando surgió el primer curso en nuestro país, y más concretamente en la Universidad Politécnica de Madrid (Muñoz y Ramió, 2013; Pérez Parras, 2016). Un buen número de organizaciones se muestran menos reacias a este movimiento y se interesan por la incorporación de estos cursos en su catálogo (Yuan y Powell, 2013; Sánchez-Vera et al., 2015).

En base a todo esto, vamos a analizar su conceptualización, sabiendo que MOOC significa curso masivo abierto en línea, y que está sujeto a unas peculiaridades, tales como que son masivos, es decir, con un número ilimitado de participantes; abierto, sin criterios de admisión y gratuitos; y en línea, llevándose a cabo en un contexto virtual (Kregor, Padgett y Brown, 2013; Yuan y Powell, 2013; Siemens, 2013; Rheingold, 2013; Castaño y Cabero, 2013; Cabero, Llorente y Vázquez Martínez, 2014; Gómez Galán, 2014; Sangrà, González-Sanmamed y Anderson, 2015).

Estos cursos se han considerado, en función de la literatura científica, como un fenómeno con un gran potencial en el mundo educativo y formativo (Martin, 2012; Cooper y Sahami, 2013; Aguaded, Vázquez Cano y Sevillano, 2013; Vázquez Cano, López Meneses y Sarasola, 2013; Yuan y Powell, 2013; Downes, 2013; López Meneses, Vázquez Cano y Román, 2015), con un gran interés mundial gracias a su formato gratuito y con accesibilidad a cualquier persona de cualquier procedencia, sin formación y sin pagar (Vázquez Cano et al., 2013; Sangrà et al., 2015). La oferta de cursos masivos demuestra la necesidad de una formación diferente y accesible y, por supuesto, de calidad (Valverde, 2014). 
Cada uno de los cursos se establece en diferentes plataformas para realizar los diferentes MOOC, siendo en España donde se desarrollan algunas, como por ejemplo, la plataforma iberoamericana MiriadaX en 2012 (Sarabia, 2016) u otras como UNED COMA (2012), UniMOOC (2013), Unx (2013) (Scopeo, 2013; Pérez Parras, 2016).

En definitiva, los MOOC están en continuo cambio, determinado por las nuevas tecnologías y las diferentes maneras de formación, que se llevarán a cabo dependiendo del contexto del sistema educativo actual y de la plataforma donde se realicen los cursos. Unos cursos que tendrán aspectos positivos y negativos, y que evidentemente es el objetivo principal de este estudio, para poder realizar una serie de propuestas de mejora que faciliten la labor de la comunidad educativa.

\subsection{Problemática de los MOOC}

Los MOOC no proporcionan una atención personalizada como puede ofrecer la enseñanza presencial, y la conversación con el estudiante se hace imposible en estos entornos: existe un problema en la comunicación, algo que no es suficiente mediante los foros o los emails. Bien es cierto, que la parte negativa de estos cursos, es que al no solicitar ni requerir ningún perfil específico, los estudiantes aumentan la tasa de abandono en cuanto logran los objetivos mínimos que solicitan dichos cursos, donde la tasa llega a ser alrededor del $90 \%$, motivado por la ausencia de horarios y de profesorado (Vivar et al., 2015). Otro estudio (Cabero, 2015) sitúa el abandono alrededor del $95 \%$.

Evidentemente, hay varios estudios que afirman que después de una semana, superada la novedad, desciende el número de estudiantes y, en algunos casos es inexistente (Lin, 2013; Sangrà et al., 2015). Con esto, algunos autores no dan importancia a ese porcentaje porque puede variar a lo largo del MOOC (Wright, 2014) o están aquellos que necesitan aprender y cuando lo hacen se dan de baja (Devlin, 2013).

Es imprescindible plantearse dónde está el problema, si son cursos bien estructurados, con contenidos atractivos o relevantes para los estudiantes y si existe coordinación entre los participantes (Suárez Guerrero y Gros, 2013; Pérez Parras, 2016). Por ello, es necesario tener una serie de características que faciliten su progreso dentro del mismo. Tales características serán la información previa del curso, nivel de compromiso del estudiante, el papel del profesorado, los recursos disponibles y su evaluación (Vázquez Cano et al., 2013; Zapata, 2013; Raposo, Martínez-Figueira y Sarmiento, 2015).

Los MOOC sugieren que el abandono escolar se relaciona más bien con la insatisfacción escolar, ya que se tratan de cursos gratuitos y con plazas ilimitadas (Liyanagunawardena, Parslow y Williams, 2014; Castaño, Maiz y Garay, 2015).

Para ubicarnos en estas debilidades de los MOOC sobre el abandono escolar, nos centraremos en dos casos (Calvo Salvador et al., 2016):

—La investigación realizada por Torres (2013) para analizar las fortalezas y debilidades vinculadas a la UNED. Dicho autor sugiere trabajar en la elaboración de módulos 
de learning analytics; mejorar los niveles de confianza y de comprobación de la identidad real en los procesos de evaluación; fomentar el uso de materiales de autoevaluación con objeto de hacer el aprendizaje más interactivo y eficiente; trabajar en el diseño de metodologías de aprendizaje disruptivas y avanzadas basadas en las dinámicas sociales y colaborativas, explotando las posibilidades brindadas por los sistemas de karma y por último, seguir trabajando por el fomento de la federación de las distintas plataformas MOOC.

-Análisis de los cursos completos de varias plataformas realizado por Ruiz Martín (2013). El autor señala que: 1. bajo el término MOOC se engloba una realidad que dista de ser homogénea, en cuanto que los cursos ofrecidos bajo estas siglas no siempre presentan las mismas características. 2. Los MOOC ofrecidos por las plataformas Coursera, EdX, Miríada X y Udacity ofrecen cursos basados en el modelo de realización de tareas y difusión del conocimiento. 3. El carácter masivo de los cursos plantea varios problemas, como el de la identificación de los alumnos o la resolución de dudas, que las plataformas deberán solucionar de cara a consolidarse como modelo educativo. 4. Los MOOC comparten la filosofía esencial del acceso libre a la información y el conocimiento. 5. El futuro de los MOOC se halla ligado al desarrollo de un modelo de negocio sostenible. Se ha visto que los cursos, como producto, son relativamente caros. 6. La oferta total de MOOC de las plataformas Coursera, EdX, Miríada X y Udacity hasta la actualidad la componen un total de 572 cursos. 7. Con el lanzamiento de los primeros cursos de Miríada X a principios de 2013 se ha consolidado el español como la segunda lengua en los MOOC, con un total de 69 cursos, lejos de los 478 que son impartidos en inglés, pero lejos también del tercer idioma, el francés, que cuenta con 12. 8. Se ha contrastado la lista de las universidades y centros de educación superior que imparten MOOC a través de estas cuatro plataformas con dos prestigiosos rankings de universidades a nivel mundial. 9. Se puede señalar que, a raíz de la información obtenida en las distintas fases de la investigación, se ha constatado que la tendencia general es que el modelo de MOOC impartidos por grandes plataformas se expanda a nivel mundial, ampliándose la oferta con cursos de universidades de otros contextos geográficos e impartidos en otras lenguas distintas al inglés.

Es importante por este motivo saber qué ofertan estos cursos en cuanto a la formación se refiere a través de Internet y cómo se puede mejorar (Aguaded, 2013; Guàrdia, Maina y Sangrá, 2013; Ramírez Fernández, 2015), además, cuál es la mejor forma de evaluación y de calidad de estos cursos como futuro de investigación, así como la importancia de conseguir más estudios sobre esta temática (Baldomero y Salmerón, 2015).

En definitiva, varios estudios plantearon la revisión de los contenidos educativos de los MOOC (Atenas, 2015; Chiappe et al., 2015; Calvo et al., 2016), para realizar una valoración crítica y reflexiva sobre las debilidades y fortalezas, limitaciones y líneas de acción futura (Calvo et al., 2016). 


\section{MATERIAL Y MÉTODOS}

Dicha investigación tiene un análisis exploratorio mixto, con un apartado cuantitativo y otro cualitativo, donde se han llevado a cabo los siguientes criterios de selección:

—Web: www.mooc.es ${ }^{1}$.

-Idiomas: todos los disponibles en este momento (inglés, español, francés, portugués, alemán y hebreo).

-Plataformas²: todas, incluidas las poco frecuentes (COURSERA, EDX, FUTURELEARN, MOOC, OPENLEARNING, entre otras).

—Análisis por categorías: con preferencia a «Educación y enseñanza».

-Categoría: «Educación y enseñanza», con los códigos (Modelos de enseñanza, herramientas, Nuevas Tecnologías, enseñanza de idiomas y otros), y donde se han analizado dentro de cada uno de los códigos: los cursos, idiomas, plataformas utilizadas, número de alumnos/as matriculados, los objetivos del curso y un apartado para observaciones.

Para analizar la parte general se utilizó una exploración cuantitativa mediante el programa SPSS (Statistical Package for the Social Sciences), en el cual se pretendía obtener los aspectos generales que dificultaban la realización de los cursos por parte del alumnado. Como resultado de este análisis se detectaron las variables relevantes que se exponen en el cuerpo del artículo.

En el gráfico 1 se ha analizado la variable «Idiomas» de todos los cursos de la página. Una variable que claramente resalta que un 93\% de estos cursos tienen predominancia por la lengua inglesa, uno de los mayores inconvenientes del alumnado para realizar cursos.

\section{IDIOMAS}

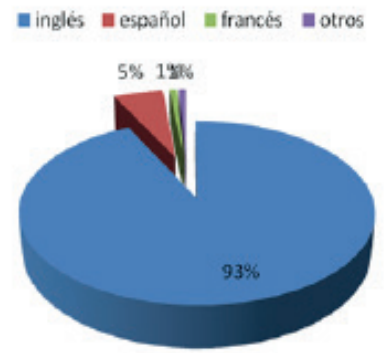

Gráfico 1. Análisis de la variable «Idiomas».

Obviamente, la base de datos de los cursos de esta página web está actualizada al 27 de enero de 2017.

2 Existen más plataformas para realizar cursos, pero en el intervalo de fechas buscado, sólo se encontraban estas disponibles en esta base de datos. 
En el gráfico 2 analizamos la variable «plataformas», donde se puede visualizar la gran cantidad de cursos que se pueden realizar a través de la plataforma COURSERA, una plataforma que está en inglés pero que la información principal está en español. A causa de esto se produce la no inscripción de los potenciales estudiantes a estos cursos.

\section{PLATAFORMAS}

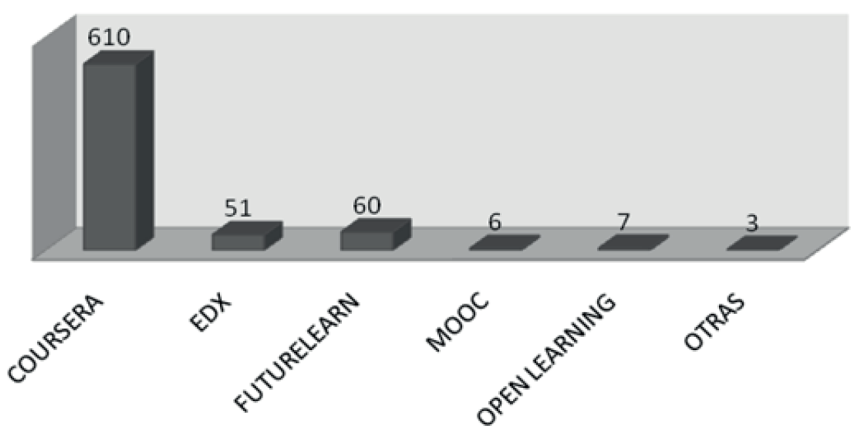

Gráfico 2. Análisis de la variable «Plataformas».

Una vez analizado estas dos varibles, se han analizado las categorías que forman parte de esta página web para determinar la elección de nuestra categoría que más se ajusta a nuestro perfil. En este caso es la de «Educación y enseñanza», que en el cómputo general solo equivale a un $6 \%$ del total (véase gráfico 3).

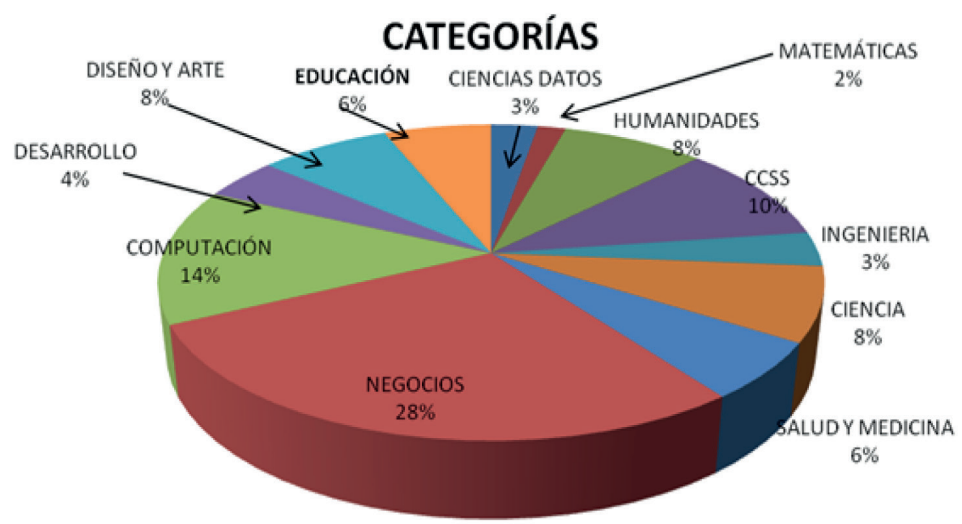

Gráfico 3. Análisis de las categorías de la web. 
Para el análisis de la categoría «Educación y enseñanza» hemos utilizado el programa de análisis cualitativo MAXQDA, donde hemos dividido dicha categoría en los siguientes códigos: «Modelos de enseñanza», «Herramientas», «NNTT»(Nuevas Tecnologías), «Enseñanza de idiomas» y «otros», realizándose un análisis del contenido del perfil del alumnado matriculado en los cursos MOOC y que han derivado en cinco subcódigos citados anteriormente. Se puede observa que hubo un porcentaje muy bajo de alumnos matriculados (véase tabla 1).

Código «Modelos de enseñanza»

\begin{tabular}{|c|c|}
\hline CURSO & $\begin{array}{l}\text { No MATRICULADOS } \\
\text { IDIOMA / PLATAFORMA }\end{array}$ \\
\hline $\mathrm{C}^{3}$ : Blended Learning: Personalizing Education for Students & $\begin{array}{l}0 \text { alumnos } \\
\text { Inglés /COURSERA }\end{array}$ \\
\hline C2: Disability Awareness and Support & $\begin{array}{l}0 \text { alumnos } \\
\text { Inglés / COURSERA }\end{array}$ \\
\hline $\begin{array}{l}\text { C3: ICT in Primary Education: Transforming children's learning across the } \\
\text { curriculum }\end{array}$ & $\begin{array}{l}0 \text { alumnos } \\
\text { Inglés / COURSERA }\end{array}$ \\
\hline C4: Learning to Teach Online & $\begin{array}{l}1 \text { alumno } \\
\text { Inglés / COURSERA }\end{array}$ \\
\hline C5: Literacy Teaching and Learning: Aims, Approaches and Pedagogies & $\begin{array}{l}0 \text { alumnos } \\
\text { Inglés / COURSERA }\end{array}$ \\
\hline C6: Performance Assessment in the Virtual Classroom & $\begin{array}{l}0 \text { alumnos } \\
\text { Inglés / COURSERA }\end{array}$ \\
\hline C7: The Place of Music in 21st Century Education & $\begin{array}{l}0 \text { alumnos } \\
\text { Inglés / COURSERA }\end{array}$ \\
\hline C8: Learning Online: Reflecting and Sharing & $\begin{array}{l}0 \text { alumnos } \\
\text { Inglés / FUTURELEARN }\end{array}$ \\
\hline C9: Academic Integrity: Values, Skills, Action & $\begin{array}{l}0 \text { alumnos } \\
\text { Inglés / FUTURELEARN }\end{array}$ \\
\hline C10: An Introduction to Evidence-Based Undergraduate STEM Teaching & $\begin{array}{l}0 \text { alumnos } \\
\text { Inglés / EDX }\end{array}$ \\
\hline C11: Aprendizaje invertido & $\begin{array}{c}6 \text { alumnos } \\
\text { Español / MOOC }\end{array}$ \\
\hline
\end{tabular}

Tabla 1. Cursos del código «Modelos de enseñanza».

3 Para codificar cada uno de los cursos y llevar a cabo su descripción se procedió a categorizar cada curso con las iniciales $\mathrm{C} 1, \mathrm{C} 2, \mathrm{C} 3$, y así sucesivamente. 
Código «Herramientas»

\begin{tabular}{|c|c|}
\hline CURSO & $\begin{array}{l}\mathbf{N}^{\circ} \text { MATRICULADOS } \\
\text { IDIOMA / PLATAFORMA }\end{array}$ \\
\hline C12: Advanced Instructional Strategies in the Virtual Classroom & $\begin{array}{c}0 \text { alumnos } \\
\text { Inglés / COURSERA }\end{array}$ \\
\hline C13: Communicating in Math in the K-12 Classroom & $\begin{array}{c}0 \text { alumnos } \\
\text { Inglés / COURSERA }\end{array}$ \\
\hline C14: Communication Skills for University Success & $\begin{array}{c}0 \text { alumnos } \\
\text { Inglés / COURSERA }\end{array}$ \\
\hline C15: Grant Proposal & $\begin{array}{c}0 \text { alumnos } \\
\text { Inglés / COURSERA }\end{array}$ \\
\hline C16: Problem-Solving Skills for University Success & $\begin{array}{c}0 \text { alumnos } \\
\text { Inglés / COURSERA }\end{array}$ \\
\hline C17: Teaching Math Through Problem-Solving K-12 & $\begin{array}{l}0 \text { alumnos } \\
\text { Inglés / COURSERA }\end{array}$ \\
\hline C18: Learning Online: Learning and Collaborating & $\begin{array}{l}\quad 0 \text { alumnos } \\
\text { Inglés / FUTURELEARN }\end{array}$ \\
\hline C19: Kickstart Your Career: Getting Ahead at University & $\begin{array}{l}0 \text { alumnos } \\
\text { Inglés / FUTURELEARN }\end{array}$ \\
\hline C20: Learning Online: Managing Your Identity & $\begin{array}{l}0 \text { alumnos } \\
\text { Inglés / FUTURELEARN }\end{array}$ \\
\hline C21: An Introduction to Evidence-Based Undergraduate STEM Teaching & $\begin{array}{l}0 \text { alumnos } \\
\text { Inglés / FUTURELEARN }\end{array}$ \\
\hline C22: Learning Online: Searching and Researching & $\begin{array}{l}0 \text { alumnos } \\
\text { Inglés / FUTURELEARN }\end{array}$ \\
\hline C23: Designing Assessments to Measure Student Outcomes & $\begin{array}{l}0 \text { alumnos } \\
\text { Inglés / FUTURELEARN }\end{array}$ \\
\hline C24: AP® Psychology - Course 5: Health and Behavior & $\begin{array}{l}0 \text { alumnos } \\
\text { Inglés / EDX }\end{array}$ \\
\hline C25: Advancing Learning Through Evidence-Based STEM Teaching & $\begin{array}{l}0 \text { alumnos } \\
\text { Inglés / EDX }\end{array}$ \\
\hline C26: College Readiness - Reading, Writing and Math & $\begin{array}{c}0 \text { alumnos } \\
\text { Inglés / MOOC }\end{array}$ \\
\hline
\end{tabular}

Tabla 2. Cursos del código «Herramientas». 


\section{Código «NNTT»}

\begin{tabular}{|c|c|}
\hline CURSO & $\begin{array}{l}\text { N}^{\circ} \text { MATRICULADOS } \\
\text { IDIOMA / PLATAFORMA }\end{array}$ \\
\hline $\begin{array}{l}\text { C27: e-Learning Ecologies: Innovative Approaches to Teaching and Learning } \\
\text { for the Digital Age }\end{array}$ & $\begin{array}{l}0 \text { alumnos } \\
\text { Inglés / COURSERA }\end{array}$ \\
\hline C28: Emerging Trends \& Technologies in the Virtual K-12 Classroom & $\begin{array}{l}0 \text { alumnos } \\
\text { Inglés / COURSERA }\end{array}$ \\
\hline C29: iMOOC102: Mastering American e-Learning & $\begin{array}{l}0 \text { alumnos } \\
\text { Inglés / COURSERA }\end{array}$ \\
\hline C30: Information \& Digital Literacy for University Success & $\begin{array}{l}0 \text { alumnos } \\
\text { Inglés / COURSERA }\end{array}$ \\
\hline C31: Integrating Technology in the K-12 Classroom & $\begin{array}{l}0 \text { alumnos } \\
\text { Inglés / COURSERA }\end{array}$ \\
\hline C32: Powerful Tools for Teaching and Learning: Digital Storytelling & $\begin{array}{l}0 \text { alumnos } \\
\text { Inglés / COURSERA }\end{array}$ \\
\hline C33: Powerful Tools for Teaching and Learning: Web 2.0 Tools & $\begin{array}{l}0 \text { alumnos } \\
\text { Inglés / FUTURELEARN }\end{array}$ \\
\hline C34: Libraries Leading with TV Whitespace & $\begin{array}{l}0 \text { alumnos } \\
\text { Inglés / MOOC }\end{array}$ \\
\hline C35: Teaching Literacy with and through Technology & $\begin{array}{l}0 \text { alumnos } \\
\text { Inglés / MOOC }\end{array}$ \\
\hline
\end{tabular}

Tabla 3. Cursos del código «NNTT».

\section{Código «Enseñanza de idiomas»}

\begin{tabular}{|l|c|}
\hline \multicolumn{1}{|c|}{ CURSO } & $\begin{array}{c}\text { No MATRICULADOS } \\
\text { IDIOMA / PLATAFORMA }\end{array}$ \\
\hline C36: English for Teaching Purposes & $\begin{array}{c}\text { alumnos } \\
\text { Inglés / COURSERA }\end{array}$ \\
\hline C37: Strategies for Teaching Perfect Tenses and Modals & 0 alumnos \\
\hline C38: Studying at Japanese Universities $/$ COURSERA
\end{tabular}

Tabla 4. Cursos del código «Enseñanza de idiomas». 


\section{Código «Otros»}

\begin{tabular}{|l|c|}
\hline \multicolumn{1}{|c|}{ CURSO } & $\begin{array}{c}\text { No MATRICULADOS } \\
\text { IDIOMA / PLATAFORMA }\end{array}$ \\
\hline C40: Fundamentals of Engineering Exam Review & $\begin{array}{c}\text { 0 alumnos } \\
\text { Inglés / COURSERA }\end{array}$ \\
\hline C41: Preparing for the AP Physics 1 Exam & $\begin{array}{c}\text { 0 alumnos } \\
\text { Inglés / COURSERA }\end{array}$ \\
\hline $\begin{array}{l}\text { C42: Preparing for an International Health Elective: Training in Global } \\
\text { Health, Ethics and Safety }\end{array}$ & $\begin{array}{c}\text { 0 alumnos } \\
\text { Inglés / FUTURELEARN }\end{array}$ \\
\hline $\begin{array}{l}\text { C43: Information Systems and Computer Applications, Part 5: Social } \\
\text { Implications }\end{array}$ & $\begin{array}{c}\text { 0 alumnos } \\
\text { Inglés / EDX }\end{array}$ \\
\hline C44: Information Systems and Computer Applications, Part 4: Programming & $\begin{array}{c}0 \text { alumnos } \\
\text { Inglés / EDX }\end{array}$ \\
\hline $\begin{array}{l}\text { C45: Information Systems and Computer Applications, Part 3: Software } \\
\text { Development }\end{array}$ & $\begin{array}{c}0 \text { alumnos } \\
\text { Inglés / EDX }\end{array}$ \\
\hline C46: Information Systems and Computer Applications, Part 2: Hardware & $\begin{array}{c}\text { alumnos } \\
\text { Inglés / EDX }\end{array}$ \\
\hline C47: Information Systems and Computer Applications, Part 1: IT & $\begin{array}{c}\text { alumnos } \\
\text { Inglés / EDX }\end{array}$ \\
\hline
\end{tabular}

Tabla 5. Cursos del código «Otros».

Una vez analizados los datos cualitativos y cuantitativos se lleva a cabo la discusión de los resultados de esta investigación.

\section{ANÁLISIS Y RESULTADOS}

Según los datos obtenidos de forma cualitativa, más del $90 \%$ de los cursos de toda la oferta se realizan en inglés, considerándose en la actualidad, uno de los problemas de los estudiantes en términos generales.

Las plataformas de acceso a estos cursos, facilitan la labor: la plataforma COURSERA, por ejemplo, está maquetada para que se pueda leer su esqueleto en español, pero el contenido sigue siendo en inglés, uno de los motivos de la creciente falta de interés del alumnado.

El perfil centrado en nuestro estudio se refiere a la categoría de «Educación y enseñanza», un código que representa al 6\% del total de todas las categorías, y que refuerza nuestra teoría en cuanto al protagonismo del idioma y de las plataformas.

Se han analizado cualitativamente cinco diferentes aspectos que profundizamos a continuación: 
- «Modelos de enseñanza»: estos cursos están enfocados a estudios sobre los distintos modelos de aprendizaje, siendo en uno de ellos, en español, donde se encuentra el mayor número de alumnos y alumnas, denominado «aprendizaje invertido», título atrayente para el alumnado, «sobre las pasiones y sus estrategias de aprendizaje» [C11]. Este apartado está muy encaminado hacia el alumnado de índole educativa, si bien es cierto que, al estar enfocados a la lengua inglesa, dificulta la matriculación de los mismos, aunque en alguno de ellos podemos encontrar a algún alumno o alumna matriculado, «consejos sobre las estrategias de aprendizaje a utilizar» [C4]. En el resto, en cambio, son cursos muy llamativos pero que a día de hoy no han obtenido matriculación alguna, bien ya sea motivado por un desconocimiento de lo que realmente significa este curso o por ignorancia. Aquí podemos citar por ejemplos cursos que tratan sobre «los modelos de aprendizaje de tres centros educativos diferentes» [C1], «métodos de educación para el acceso de personas con discapacidad» [C2], «historias sobre las escuelas del mundo» [C3], entre otras. Son muchas las ventajas que ofrecen estos cursos: más conocimientos, otra forma de acceder al contenido, flexibilidad horaria, pero su barrera lingüística hace de esto un paso atrás como comentaremos más adelante.

— «Herramientas»: este código está representado por aquellas habilidades o características que facilitan la labor del estudiante o del profesorado. En este caso, todos los cursos presentados en esta página web son de habla inglesa, y con varias plataformas de contenido únicamente en inglés, lo que dificulta sobremanera la matriculación en los mismos. Es el caso de cursos como «trabajar la comunicación en línea con otros» [C18], «técnicas para comenzar la Universidad bien» [C19] o por ejemplo «habilidades de trabajo en línea y ser capaz de analizar críticamente las fuentes de información» [C22] favorecen las técnicas para poder desenvolverse en la vida, pero son cursos que al ser nombrados en otro idioma dificultan su percepción.

— «NNT »: el siguiente código lógicamente tiene la ventaja que llama la atención desde un primer momento, por incluir dentro de su temática principal a las nuevas tecnologías. Unas nuevas tecnologías asociadas a la educación y a la disrupción educativa de las MOOC. Desafortunadamente, hasta el momento no se ha matriculado ningún alumno o alumna, a pesar de ser una materia muy atrayente, como son los casos de los cursos sobre «la incorporación de las NNTT sobre la educación tradicional» [C27], «integrar una variedad de recursos tecnológicos» [C31], «crear una historia digital con un simple guión y una sola imagen» [C32] o «preparación a los profesores para combinar textos tradicionales y medios educativos como los libros electrónicos» [C35]. En este último caso tiene el inconveniente que, además de ser un curso de habla inglesa con una plataforma inglesa, está destinado para profesionales del sector de la educación, por tanto, otra nueva barrera que nos encontramos aquí.

— «Enseñanza de idiomas»: en la sociedad de hoy en día, conocer diferentes idiomas en un sinónimo de acercamiento a otras culturas y contextos. Desde este punto de vista, es una ventaja familiarizarse con otros idiomas, pero un claro inconveniente si se realiza partiendo de un idioma desconocido. Varios cursos se pueden realizar sobre 
distintas temáticas como sobre «inglés y con una metodología didáctica que pretende ayudar a los profesores universitarios a hacer su enseñanza en inglés» [C36], con un alto nivel de inglés exigido, «de acceso al estudio de universidades japonesas» [C38] o «habilidades de lectura en inglés» [C39].

— «Otros»: en este caso se agrupan aquellos cursos que por temática no corresponden a ninguno de los casos anteriores, y no están muy ligados a la categoría analizada. Son cursos de diferentes temáticas que tienen a la enseñanza como denominador común pero que deberían de ir en otra sección. Por lo tanto, es un inconveniente claro de no matriculación del alumnado, ya sea motivado por su posición en esta página o la dificultad para poder realizarlo. Podemos encontrar cursos tales como «material sobre el examen de Ingeniería» [C40], «preparación del examen de Física» [C41] o «el examen CLEP de Sistemas de Información y Aplicaciones de Computadora» [C43-C47].

En definitiva, una serie de ventajas e inconvenientes que hemos podido observar, y que resaltan los aspectos sobre una serie de propuestas de mejora, que en general, toda página web y plataformas deberían realizar para aumentar el número del alumnado matriculado en estos cursos.

\section{DISCUSIÓNY CONCLUSIONES}

La llegada de las nuevas tecnologías a nuestra sociedad fue una revolución necesaria por la exigencia de las distintas compañías y empresas. Una tecnología que se introdujo poco a poco en la enseñanza tradicional, y que hoy en día es una herramienta imprescindible para llevar a cabo ciertas actividades de enseñanza. Tanto es así, que hace menos de dos lustros, esta enseñanza derivó a lo que conocemos como MOOC.

Unos MOOC que se ubican en una serie páginas web, muy fáciles de acceder y de encontrar, con una serie de cursos a disposición del usuario, catalogados en función de una temática, con puntualizaciones sobre datos de cada uno de los cursos (fechas, objetivos, duración, etc.), y con el acceso a una serie de plataformas que mejoran la labor de estas páginas web, maquetadas para que el recorrido durante ella sea más cómodo. Unas plataformas que están enfocadas a uno de los idiomas universales por excelencia, el inglés, uno de los requisitos imprescindibles en la sociedad multicultural que en la actualidad estamos viviendo.

Una falta de interés entre los estudiantes motivado, sin lugar a dudas, por ser cursos gratuitos y con acceso para todo el alumnado, con independencia de su nivel de idioma, un nivel que, si bien es cierto, en algunos de los cursos si es un requisito marcado por los mismos, pero que al ser escrito en inglés no es del todo entendible por la mayoría de los alumnos y alumnas que acceden a este tipo de páginas.

Es verdad que las plataformas donde se realizan los cursos mejoran el acercamiento al alumnado, de ahí que éste pueda matricularse con facilidad, pero cuando comienzan el curso, su falta de interés es creciente por desconocimiento del idioma o de la terminología específica del mismo. 
Otros cursos están destinados a especialistas de una determinada profesión, que ya sea por motivos de desconocimiento de la ubicación del mismo o por no saber el idioma del curso, se convierte en un hándicap.

Por todo ello, se han formulado una serie de propuestas de mejora para que aumente la tasa de matriculación con respecto a años anteriores. Las propuestas son las siguientes:

1. Diseño de la página web: en términos generales los cursos deben estar divididos por categorías, y especificadas en función del idioma principal, con posibilidad de convertirlo al idioma deseado. Aquí los cursos deben tener como temática principal la categoría que se estipule, pero es conveniente hacer una selección crítica de los mismos.

2. Plataformas: como en el caso anterior, deben estar con su idioma original, pero con la posibilidad también de transformarlo en el idioma que cada uno desee. Estas plataformas deben guiar al alumnado en todo momento en su aprendizaje, para que el alumnado no pierda interés por los mismos.

3. Idiomas: debe haber una sección en los mismos para visualizar mejor el contenido por cada uno de los usuarios, con posibilidad de elección múltiple.

4. Sistema de evaluación: mejorar herramientas de evaluación de estos cursos, para ello es conveniente adaptarlos en función del tipo de cursos, de los objetivos que se pretendan, etc. Es importante aquí la autoevaluación, entre el mismo alumnado y de las plataformas que realizan los cursos.

5. Chats: creación de un chat para poder recibir críticas constructivas al instante, así como para potenciar el feedback y mantener al alumnado motivado con el curso.

La disrupción educativa de los MOOC es evidente, y está en continuo auge, gracias a la ayuda de los diferentes responsables de cada uno de los cursos, plataformas, usuarios, etc. La triangulación existente entre el profesorado, usuario y plataforma debe de tener una serie de requisitos indispensables para que la marcha del mismo sea productiva y aumente el número de alumnos y alumnas matriculados.

\section{REFERENCIAS BIBLIOGRÁFICAS}

Aguaded, I. (2013). La revolución MOOC, ¿una nueva educación desde el paradigma tecnológico? [The MOOC Revolution: A New Form of Education from the Technological Paradigm?]. Comunicar, XXI(41), 07-08. DOI: https://doi.org/10.3916/C41-2013-a1

Aguaded, I., Vázquez Cano, E. y López Meneses, E. (2016). El impacto bibliométrico del movimiento MOOC en la Comunidad Científica Española. Educación XX1, 19(2), 77-104. DOI: https://doi.org/10.5944/educxx1.16454

Aguaded, I., Vázquez Cano, E. y Sevillano, M. L. (2013). MOOC, ¿turbo capitalismo de redes o altruismo educativo? Hacia un modelo más sostenible. En Scopeo Informe, 2 (Eds.), 
MOOC: Estado de la situación actual, posibilidades, retos y futuro. Salamanca: Universidad de Salamanca. Recuperado de http://scopeo.usal.es/wp-content/uploads/2013/06/ scopeoi002.pdf [Consulta: 10-01-2017].

Anderson, T. (2013). Promise and/or Peril: MOOC and Open and Distance Education. Commonwealth of Learning. Athabasca: Athabasca University. Recuperado de http://citeseerx.ist.psu.edu/viewdoc/download?doi=10.1.1.363.4943\&rep=rep1\&type=pdf [Consulta: 20-12-2016].

Atenas, J. (2015). Modelo de democratización de los contenidos albergados en los MOOC. RUSC. Universities and Knowledge Society Journal, 12(1), 3-14. DOI: https://doi. org/10.7238/rusc.v12i1.2031

Baldomero, M. y Salmerón, J. L. (2015). EduTool®: Un instrumento para la evaluación y acreditación de la calidad de los MOOC. Educación XX1, 18(2), 97-123. DOI: https://doi. org/10.5944/educxx1.13233

Baraniuk, R. G. (2007). Challenges and Opportunities for the Open Education Movement: A Connexions Case Study. En T. Liyoshi y M. S. Vijay (Eds.), Opening up Education: The Collective Advancement of Education through Open Technology, Open Content, and Open Knowledge (pp. 116-132). Cambridge: MIT Press.

Cabero, J. (2015). Visiones educativas sobre los MOOC. RIED. Revista Iberoamericana de Educación a Distancia, 18(2), 39-60. DOI: https://doi.org/10.5944/ried.18.2.13718

Cabero, J., Llorente, M. C. y Vázquez Martínez, A. I. (2014). Las tipologías de MOOC: su diseño e implicaciones educativas. Revista de currículum y formación del profesorado, 18(1), 13-26. Recuperado de https://www.ugr.es/ recfpro/rev181ART1.pdf

Calvo Salvador, M. A., Rodríguez Hoyos, C. y Fernández Díaz, E. M. (2016). ¿Cómo son los MOOC sobre educación? Un análisis de cursos de temática pedagógica que ofertan en castellano. Digital Education Review, (29), 298-319. Recuperado de https://revistes. ub.edu/index.php/der/article/view/14680

Castaño, C. y Cabero, J. (2013). Enseñar y aprender en entornos m-learning. Madrid: Síntesis.

Castaño, C., Maiz, I. y Garay, U. (2015). Diseño, motivación y rendimiento en un curso MOOC cooperativo [Design, Motivation and Performance in a Cooperative MOOC Course]. Comunicar, XXII(44), 19-26. DOI: https://doi.org/10.3916/C44-2015-02

Chiappe, A., Hine, N. y Martínez Silva, N. A. (2015). Literatura y práctica: una revisión crítica acerca de los MOOC [Literature and Practice: A Critical Review of MOOC]. Comunicar, XXII(44), 9-18. DOI: https://doi.org/10.3916/C44-2015-01

Conole, G. (2013). Designing for learning in an open world. New York: Springer. DOI: https:// doi.org/10.1007/978-1-4419-8517-0

Cooper, S. y Sahami, M. (2013). Education Reflections on Stanford's MOOC: New Possibilities in Online Education Create New Challenges. Communications of the ACM, 56(2), 28-30. DOI: https://doi.org/10.1145/2408776.2408787

Dans, E. (2009). Online Education: Educational Platforms and the Openness Dilemm. RUSC, 6(1), 22-30. DOI: https://doi.org/10.7238/rusc.v6i1.26

D’Antoni, S. (2009). Open Educational Resources: Reviewing Initiatives and Issues. Open Learning, 24(1), 3-10. https://doi.org/10.1080/02680510802625443 
Devlin, K. (2013). MOOC and the Myths of Dropout Rates and Certification. The Huffington Post. Recuperado de https://www.huffpost.com/entry/moocs-and-the-myths-ofdr_b_2785808?guccounter=1 [Consulta: 23-12-2016].

Downes, S. (2013). MOOC-The Resurgence of Community in Online Learning. Half an Hour. Recuperado de https://halfanhour.blogspot.com/2013/05/mooc-resurgence-of-community-in-online.html [Consulta: 26-11-2016].

Earl, K. (2013). Student views on short-text assignment formats in fully online courses. Distance Education, 34(2), 161-174. DOI: https://doi.org/10.1080/01587919.2013.793639

Gaebel, M. (2013). MOOC, Massive Open Online Courses. EUA Occasional Papers. Bruselas: European University Association. Recuperado de http://supporthere.org/sites/default/ files/eua_occasional_papers_moocs_4.pdf [Consulta: 12-12-2016].

Gallego-Arrufat, M. J., Gamiz-Sánchez, V. M. y Gutiérrez-Santiuste, E. (2015). Tendencias en la evaluación del aprendizaje en cursos en línea masivos y abiertos. Educación XX1, 18(2), 77-96. DOI: https://doi.org/10.5944/educxx1.12935

Gómez Galán, J. (2014). El fenómeno MOOC y la universidad de la cultura: las nuevas fronteras de la educación superior. Revista de Currículum y Formación del Profesorado, 18(1), 73-91. Recuperado de https://recyt.fecyt.es/index.php/profesorado/article/view/41069

Grossman, D. (2013). Massive open online courses - threat or opportunity? BBC News. Recuperado de https://www.bbc.com/news/education-23069542 [Consulta: 20-12-2016].

Guárdia, L., Maina, M. y Sangrá, A. (2013). MOOC Design Principles. A Pedagogical Approach from the Learner's Perspective. eLearning Papers, (33). Recuperado de http:// openaccess.uoc.edu/webapps/o2/bitstream/10609/41681/1/In-depth_33_4\%282\%29.pdf

Jung, I. (2011). The Dimensions of e-learning Quality: From the Learner's Perspective. Educational Technology Research and Development, 59(4), 445-464. DOI: https://doi. org/10.1007/s11423-010-9171-4

Kregor, G., Padgett, L. y Brown, N. (2013). Technology Enhanced Learning and Teaching. Hobart: Tasmanian Institute of Learning and Teaching, University of Tasmania.

Lane, L. (2012). Three Kinds of MOOC. Lisa's (online). Teaching \& History Blog. Recuperado de http://lisahistory.net/wordpress/musings/three-kinds-of-moocs [Consulta: 15-8-2012].

Lin, M. (2013). Case study: Low participation rate raises question about effectiveness of Massive Open Online Course (MOOC). MulinBlog. Recuperado de http://www.mulinblog. $\mathrm{com} /$ case-study-low-participation-rate-raises-question-about-effectiveness-of-massiveopen-online-course-mooc [Consulta: 7-12-2016].

Little, G. (2013). Massively Open? The Journal of Academic Librarianship, 39(3), 308-309. DOI: https://doi.org/10.1016/j.acalib.2013.03.004

Liyanagunawardena, T. R., Parslow, P. y Williams, S. A. (2014). Dropout: MOOC Participants' Perspective. In U. Crees y C. Delgado (Eds.), Proceedings of the European MOOC Stakeholder Summit 2014, (pp. 95-100). Reading: University of Reading.

López Meneses, E., Vázquez Cano, E. y Román, P. (2015). Análisis e implicaciones del impacto del movimiento MOOC en la comunidad científica: JCR y Scopus (2010-13) [Analysis and Implications of the Impact of MOOC Movement in the Scientific Community: JCR 
and Scopus (2010-13)]. Comunicar, XXII(44), 73-80. DOI: https://doi.org/10.3916/C442015-08

Luján, S. (2013). De la clase magistral al MOOC: doce años de evolución de una asignatura sobre programación de aplicaciones web. Revista de Docencia Universitaria, (11), 279-300. DOI: https://doi.org/10.4995/redu.2013.5557

Marcelo, C. y Zapata, M. (2008). Evaluación de la calidad para programas completos de formación docente a través de estrategias de aprendizaje abierto y a distancia. RED, VII, 1-6. Recuperado de https://revistas.um.es/red/article/view/125191

Martin, F. G. (2012). Will Massive Open Online Courses Change how we Teach? Communications of the ACM, 55(8), 26-28. DOI: https://doi.org/10.1145/2240236.2240246

Muñoz, A. y Ramió, J. (2013). Cryt4you y la utilidad de los MOOC en la formación online en lengua española. Innovación educativa, (23), 231-240. Recuperado de https://revistas.usc. gal/index.php/ie/article/view/1072

Pappano, L. (2012). Year of the MOOC. The New York Times. Recuperado de https://www. nytimes.com/2012/11/04/education/edlife/massive-open-online-courses-are-multiplyingat-a-rapid-pace.html?pagewanted=all\&_r=0Pappano, +2012 [Consulta: 1-12-2016].

Pérez Parras, J. (2016). Nuevas tecnologías e influencia del ambiente dentro del proceso enseñanza-aprendizaje: Impacto de los cursos MOOC en educación. International Journal of Educational Research and Innovation (IJERI), (6), 176-186. Recuperado de https:// www.upo.es/revistas/index.php/IJERI/article/view/1635

Ramírez Fernández, M. B. (2015). Propuesta de certificación de calidad de la oferta española educativa de cursos MOOC. International Journal of Educational Research and Innovation (IJERI), (3), 121-133. Recuperado de https:/www.upo.es/revistas/index.php/ IJERI/article/view/1448

Raposo, M., Martínez-Figueira, E. y Sarmiento, J.A. (2015). Un estudio sobre los componentes pedagógicos de los cursos online masivos [A Study on the Pedagogical Components of Massive Online Courses]. Comunicar, XXII(44), 27-36. DOI: https://doi.org/10.3916/ C44-2015-03

Rheingold, H. (2013). MOOC, Hype, and the Precarious State of Higher Ed: Futurist Bryan Alexander. Dmlcentral. Recuperado de https://clalliance.org/blog/moocs-hype-and-the-precarious-state-of-higher-ed-futurist-bryan-alexander/ [Consulta: 12-11-2016].

Ruiz Martín, P. (2013). Presente y futuro de los Massive Open Online Courses (MOOC). Análisis de la oferta completa de cursos de las plataformas COURSERA, EdX, MIRÍADA X y UDACITY. Trabajo Fin de Máster. Madrid: Universidad Complutense de Madrid. Recuperado de http://eprints.ucm.es/23502/2/MOOCs.pdf

Sánchez-Vera, M. M., León-Urrutia, M. y Davis, H. (2015). Desafíos en la creación, desarrollo e implementación de los MOOC: El curso de Web Science en la Universidad de Southampton [Challenges in the Creation, Development and Implementation of MOOC: Web Science Course at the University of Southampton]. Comunicar, XXII(44), 37-44. DOI: https://doi.org/10.3916/C44-2015-04 
Sancho, T., Oliver, M. y Gisbert, M. (2015). MOOC en Cataluña: Un instrumento para la innovación en educación superior. Educación XX1, 18(2), 125-146. DOI: https://doi. org/10.5944/educxx1.13462

Sangrá, A., González-Sanmamed, M. y Anderson, T. (2015). Metaanálisis de la investigación sobre MOOC en el período 2013-2014. Educación XX1, 18(2), 21-49. DOI: https://doi. org/10.5944/educxx1.13463

Sarabia, C. M. (2016). Nuevas culturas educativas: los MOOC en las universidades españolas. C\&E. Cultura y Educación, 28(1), 196-212. DOI: https://doi.org/10.1080/11356405.201 5.1120451

Scopeo (2013). MOOC: Estado de la situación actual, posibilidades, retos y futuro. Scopeo Informe, 2. Salamanca: Universidad de Salamanca. Recuperado de http://scopeo.usal. es/wp-content/uploads/2013/06/scopeoi002.pdf [Consulta: 14-11-2016].

Siemens, G. (2013). Massive Open Online Courses: Innovation in Education? En R. McGreal, W. Kinuthia y S. Marshall (Eds.), Open Educational Resources: Innovation, Research and Practice (pp. 5-15). Vancouver: Commonwealth of Learning - Athabasca University.

Silva, I. (2014). Utilización de MOOC en la formación docente: ventajas, desventajas y peligros. Revista de currículum y formación del profesorado, 18(1), 155-166. Recuperado de https://recyt.fecyt.es/index.php/profesorado/article/view/41073

Suárez Guerrero, C. y Gros, B. (2013). Aprender en red: de la interacción a la colaboración. Barcelona: Editorial UOC.

Torres, D. (2013). Reflexiones y primeros resultados de MOOC en Iberoamérica: UNEDCOMA Y UNX. Campus virtuales, 2(1), 64-73. Recuperado de http://uajournals.com/ojs/ index.php/campusvirtuales/article/view/29

Valverde, J. (2014). MOOC: una visión crítica desde las ciencias de la educación. Revista de currículum y formación del profesorado, 18(1), 93-111. Recuperado de https://www.ugr. es/ recfpro/rev181ART6.pdf

Vázquez Cano, E., López Meneses, E. y Sarasola, J. L. (2013). La expansión del conocimiento en abierto: Los MOOC. Barcelona: Octaedro.

Vivar, H., Vinader, R. y Abuín, N. (2015). Los MOOC en la enseñanza superior: una tarea pendiente. Estudios sobre el Mensaje Periodístico, 21(1). DOI: https://doi.org/10.5209/ rev_ESMP.2015.v21.n1.49115

Wright, R. (2014). The Death of MOOC has been greatly exaggerated. Slate. Recuperado de https://slate.com/technology/2014/04/mooc-completion-rates-dont-matter.html [Recuperado de 29-12-2016].

Yuan, L. y Powell, S. (2013). MOOC and Open Education: Implications for Higher Education. Bolton (UK): JISC CETIS Publications-University of Bolton. Recuperado de https://espace.mmu.ac.uk/619735/1/MOOCs-and-Open-Education.pdf [Consulta: 22-12-2016].

Zapata, M. (2013). MOOC, una visión crítica y una alternativa complementaria: La individualización del aprendizaje y de la ayuda pedagógica. Campus Virtuales, 2(1), 20-38. Recuperado de http://www.uajournals.com/campusvirtuales/journal/2/2.pdf 